\title{
Performance of Wiener Filter and Adaptive Filter for Noise Cancellation in Real-Time Environment
}

\author{
G.V.P.Chandra Sekhar \\ Yadav \\ Student, M.Tech, DECS \\ Gudlavalleru Engineering \\ College \\ Gudlavalleru-521356, Krishna \\ District \\ Andhra Pradesh, India
}

\author{
B. Ananda Krishna, Ph.D \\ Professor, Department of ECE \\ Gudlavalleru Engineering \\ College \\ Gudlavalleru-521356, Krishna \\ District \\ Andhra Pradesh, India
}

\author{
M. Kamaraju, Ph.D \\ Professor, Head of Department \\ ECE \\ Gudlavalleru Engineering \\ College \\ Gudlavalleru-521356, Krishna \\ District \\ Andhra Pradesh, India
}

\begin{abstract}
Form the past several decades' noise cancellation in speech signal gains researchers' attention. Several techniques were developed for noise cancellation among them optimal wiener filter can be the one of the most fundamental approach for noise cancellation. Later on adaptive filter was introduced to attain better performance. This paper shows the capacity of wiener filter and adaptive filter for removal of noise by estimating the signal by means of removing the noise signal form the corrupted signal. Wiener filter plays a central role in wide range of applications such as linear prediction, echo cancellation, signal restoration, channel equalization and system identification. In this paper the performance of wiener filter and adaptive filter for removal of noise in the presence of real time environment are compared. In the existing papers the authors have proposed the theory of wiener filter and adaptive filter algorithms in real time environment like recorded speech. So this is paper is going to take the part of the existing paper and going to perform the noise cancellation. In order to measure the performance step size is the main factor for the convergence speed and mean square error. Wiener filter provides better performance for noise cancellation but it requires large no. of computations i.e., complexity and cost of the system is going to increase, so adaptive filter is the alternate approach for removal of noise with moderate complexity and cost. The simulation result clearly shows that wiener filter gives the better performance but due to high cost adaptive filter is the choice of many applications. This paper is going to discuss about wiener filter theory, wiener filter problem, solution to optimal filtering, adaptive filtering, adaptive algorithm, study of wiener filter and adaptive filter for noise reduction etc.
\end{abstract}

\section{Keywords}

Wiener filter, optimum linear filters, Adaptive filter,Noise cancellation,Performance comparison, Convergence speed, Minimum mean squared error, Least mean square

\section{INTRODUCTION}

Wiener filters are the class of optimal linear filters which involves linear estimation of the desired signal by comparing the noise signal with the desired signal corrupted by noise. Wiener proposed an optimal solution to the problem of noise cancellation but it has some practical limitations. Adaptive filters are the digital filters with an impulse response, or transfer function that can be modified time to time to match with the characteristics of the desired system. Adaptive filters are used in many applications because of its adapting nature according to the input signal. Noise is the signal that mixes up with the desired signal and corrupts it in any type of communication system. In signal processing adaptive filters are the alternate method for recovering desired speech from the noise. Several algorithms have been proposed in earlier days to detect the desired signal. Least mean square (LMS) algorithm was the most efficient method in terms of computation and storage requirements but it has low convergence speed. The paper consists of objective, existing work, proposed work, parameters to be compared, filtering,need for adaptive filtering, adaptive algorithms, real time environment, simulation results, conclusion and future scope and references.

\section{OBJECTIVE}

The main objective of the project is to remove the real time noise that is present within the signal and compare the performance of wiener filter and adaptive filter for noise cancellation in real time environment.

\section{EXISTING WORK}

The authors Yen-Hsiang Chen et al [1] proposed, "An Automotive Application of Real-time Adaptive Wiener Filter for Non-Stationary Noise Cancellation in a Car Environment".

In this paper, a real-time adaptive wiener filter with two micro phones is implemented to reduce noisy speech when noise signals and desired speech are incoming simultaneously. This paper also gives an analysis of different matrix sizes of the wiener filter so as to enable the possibility of real-time implementation. The performance of the proposed design is measured by as much as $20 \mathrm{~dB}$ noise reduction, and the proposed adaptive wiener matrix update speed achieves a 28.6 $\mathrm{ms} /$ frame, with a matrix size of 200 .

The authors H.kaur et al [2] proposed, "Performance and Convergence Analysis of LMS Algorithm".

In this paper, Instead of computing $W_{\text {opt }}$ as suggested by Wiener-Hopf equation, in LMS the coefficients are adjusted from sample to sample in such a way as to minimize the MSE. The LMS algorithm is based on steepest descent algorithm. This paper also gives the analysis of LMS algorithm for different input samples and different number of iterations. The simplicity of the LMS algorithm and ease of implementation make it first choice in many applications. The convergence rate is low for this algorithm.

The authors Talwar.R et al [3] proposed "Performance 
comparison of adaptive algorithms for noise cancellation".

In this paper the authors have compared the performance of different adaptive algorithms like LMS, NLMS, and RLS algorithms for sinusoidal input. Performance is compared for different step size and different number of iterations. Three adaptive filter algorithms have been compared by simulation to achieve high convergence rate and minimum mean square error with noise and different values of $\mu$. Every algorithm works on different methods for noise cancellation and improves system performance.

The authors G.V.P.Chandra Sekhar Yadav et al [4] proposed "Study of different adaptive filter algorithms for noise cancellation in real time environment".

In this paper the authors have proposed the performance of wiener filter and different adaptive filter algorithms like LMS, NLMS and RLS algorithms for noise cancellation in real time environment like recorded speech as the input and different noise signals are added to it and then desired signal is estimated by using the adaptive algorithms.

\section{PROPOSED WORK}

In the existing work, the authors G.V.P.Chandra Sekhar Yadav and Dr. B. Ananda Krishna [4] proposed the performance of wiener filter and adaptive filter algorithms like LMS, NLMS and RLS algorithms in real time environment. Now, this paper is going to work on the part of the existing work like wiener filter and adaptive filter algorithm i.e. LMS algorithm in real time environment by taking input as recorded speech with different background noises to compare their performance and the performance is analyzed with different lengths and parameters of the input as shown in figure 1 .

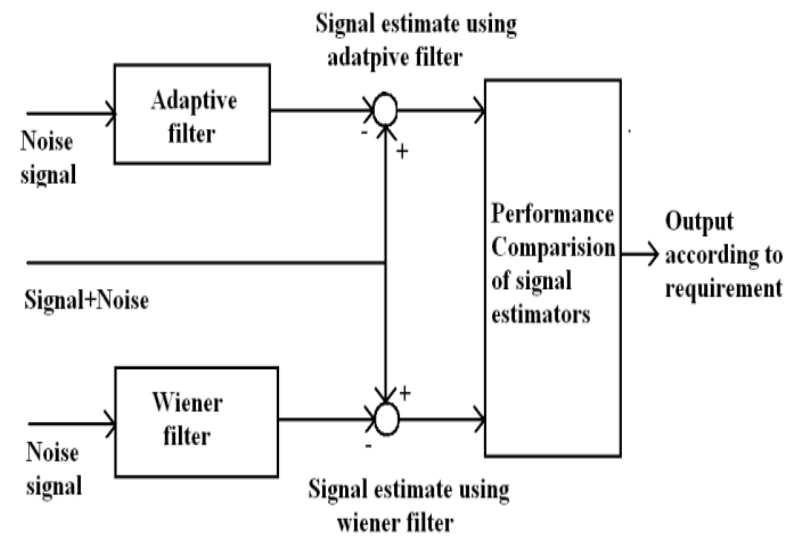

Fig 1: Block diagram of proposed work

\section{PARAMETERS}

Performance of the systems can be measured with respect to several parameters like Minimum mean square error, convergence speed, computational complexity, stability, robustness, signal to noise ratio and filter length. Minimum mean square error denotes how the system will adapt to a given solution. Small MSE indicates that the system is accurately modified or adapted to a solution for the system. Large MSE indicates the system can't be adapted according to the requirement. Step size is the major factor for convergence speed. Convergence rate indicates the rate at which the filter converges to its resultant state.Usually faster convergence rate is the desired characteristic of the adaptive filter. Computational complexity is important particularly in real time adaptive applications. Robustness is how the system can resist both desire signal and noise signal. The filter length of the adaptive system is inherently tied to many of the other performance measures. The length of the filter specifies how accurately a given system can be modeled by the adaptive filter. In addition, the filter length affects the convergence rate, by increasing or decreasing computation time, it can affect the stability of the system, at certain step sizes, and it affects the minimum MSE.

\section{FILTERS}

Filters are the basic elements in the signal processing system. Filter is a device used to suppress the unwanted signal i.e. noise from the desired signal as shown in figure 2and there are several techniques are used for filtering. Usual method of estimating the signal corrupted by noise is to pass to it through the filter that tend to suppress the noise and leaving the desired signal this is so called direct filtering. In general filters are of two types. Fixed filters and adaptive filters

In fixed filters the frequency response or filter coefficients of the filters are fixed and it requires prior knowledge of the input signals. If the signal and noise characteristics are known beforehand then it is easy to design the filter that passes the frequencies contained in the signal and rejects the frequency band occupied by noise.

An adaptive filter on the other hand requires less or no knowledge about the signal and noise. In adaptive filters the filter coefficients are adjustable or modifiable.

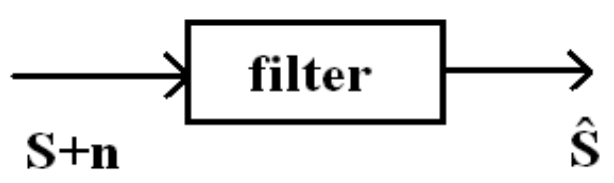

Fig 2: Basic filter

\subsubsection{Basic wiener filter theory}

Wiener filter is a filtering system that is an optimal solution to the statistical filtering problem [1].In the statistical approach to the solution of the linear filtering problem we have to assume the characteristics of the desired signal to be estimated and noise to be removed. So the filter has to be designed in such a way that the output of filter minimizes the effect of the noise from the noisy data which is given as input to the filter [11].

The meaningful approach to the problem of linear filtering is by minimizing the mean square value of error signal which is described as the difference between the desired signal and filter output. The mean square error (MSE), $\xi$, is obtained by taking the "expectation" of the squareof the error signal $\left(\mathrm{e}_{\mathrm{k}}\right)$.

For stationary inputs the solution to this problem is commonly a wiener filter. Many adaptive algorithms can be viewed as approximations of the discrete wiener filter. The signals $x_{k}$ and $y_{k}$ which are noise and noisy signals respectively are applied to the filter as shown in figure 3. The noisy signal consists of the desired data and noise, then wiener filter gives the output as the estimate of the noise signal which is then subtracted from the noisy data to yield error $\left(e_{k}\right)$ signal. 


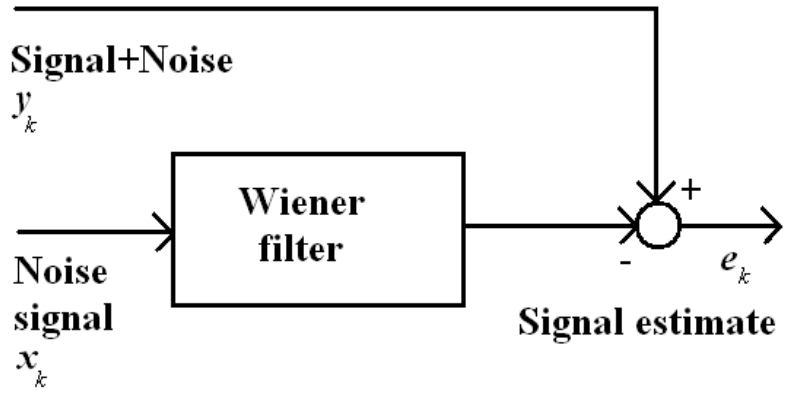

Fig 3: Wiener filter

$$
e_{k}=y_{k}-\widehat{n_{k}}=y_{k}-\sum_{i=0}^{N-1} w(i) \cdot x_{k-i}
$$

Where $x_{k}$ and $w$ are the input signal vector and weight vector respectively of the filter and are given by

$$
\begin{gathered}
x_{k}=\left[x_{k} x_{k-1} x_{k-2} x_{k-3} \ldots \ldots x_{k-(N-1)}\right]^{T} \\
w=[w(0) w(1) w(2) w(3) \ldots \ldots . w(N-1)]^{T}
\end{gathered}
$$

Wiener filter is represented by coefficient vectorw. Wiener filter coefficients are calculated to minimize the mean square value between the filter output and desired signal. Error signal is obtained by taking the difference between the noisy signal and estimation of the noise signal, after that mean square error (MSE) is calculated by taking expectation to square of the error signal $e_{k}^{2}$.

$$
\xi=\in\left\{e_{k}^{2}\right\}=\in\left\{y_{k}^{2}\right\}-2 W^{T} R_{Y X}+W^{T} R_{X X} W
$$

Performance surface is used to determine the solution to the statistical filtering problem i.e. it finds the optimum value of the filter coefficients where the mean square error should be minimized [5].

\subsubsection{Performance surface}

The mean square error of the wiener filter is a quadratic function of filter coefficient and has single minimum value as mentioned above. A plot of mean square erroragainst the filter coefficient $W$ is bowl shaped surface with a unique bottom point as shown in figure 4. It is known as the mean square error surface and is Non-negative. The gradient of the mean square error surface is given by

$$
\nabla=\frac{d \xi}{d w}=0-2 P+2 R W
$$

Where $P=R_{Y X}$ called as toeplitz matrix andis the N-length cross correlation vector which is symmetric

$R=R_{X X}$ is the $N \times N$ auto correlation matrix.

The least mean square error point corresponds to the minimum error power. At this operating point the mean square error surface has zero gradient value and filter weight vector has its optimum value $W_{\text {opt }}$.

$$
\begin{gathered}
\nabla=0 \\
-2 P+2 R W=0 \\
2 P=2 R W \\
W_{\text {opt }}=R^{-1} P
\end{gathered}
$$

This is known as the wiener-Hopf equation or solution [1]. In adaptive filter it adapts or adjusts the filter weights $w(0) w(1) w(2) w(3) \ldots \ldots$ according to suitable algorithm, to find the optimum value of the weight vector on the mean square error surface.

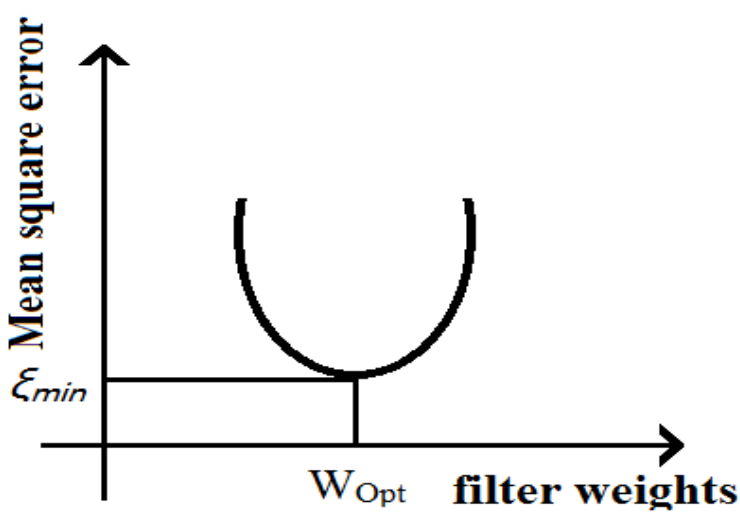

Fig 4: Mean square error surface

Wiener filter is formulated to map an input signal to the output that is as close to the desired signal as possible.

A signal is completely recoverable from noise when the spectra of the signal and noise don't overlap each other. If the signal and noise occupies different parts of the frequency spectrum, they can be separated by using either low pass or high pass filter. If the signal and noise has overlap spectra, in this case it is not possible to completely separate the signal from noise but the effects of noise can be reduced by using wiener filter.

The choice of wiener filter order affects:

The ability of the filter to remove the distortion and reduce the noise.

The computational complexity of the filter and

The numerical stability of the wiener solution.

And also the wiener filter has a limited practical usefulness because of the following reasons.

It requires the auto correlation matrix $\mathrm{R}$ and the cross correlation vector $\mathrm{P}$ both of which are not known. It involves matrix inversion, which is time consuming. If the signals are non-stationary then both $\mathrm{R}$ and $\mathrm{P}$ will change with time and so $W_{o p t}$ will have to be computed repeatedly.

For real time applications, a way of obtaining $W_{\text {opt }}$ on a sample by sample basis is required. Adaptive algorithms are used to achieve this without having to compute $\mathrm{R}$ and $\mathrm{P}$ explicitly or performing a matrix inversion. The solution to Wiener-Hopf equation is the steepest descent algorithm [6].

\subsubsection{Adaptive filters}

Adaptive filter is essentially a digital filer with self-adjusting characteristics. It adapts automatically to changes in its input signals [6]. Adaptive filters have the capability of adaptively tracking the signal under non stationary conditions.

In the basic form, the wiener theory assumes that signals are stationary. However if the coefficients are periodically regulated for every $\mathrm{N}$ samples then the filter adapts itself to the characteristics of the signal become an adaptive filter. Adaptive filter provides alternate solution to the formulation of wiener filter problem [8].

Adaptive filters are used in the following cases:-

When it is necessary for the filter characteristics to be variable, adapted to changing conditions.

When there is spectral overlap between signal and noise.

If the band occupied by the noise is unknown or varies with time. 
The use of conventional filters in the above cases would lead to unacceptable distortion of the desired signal.

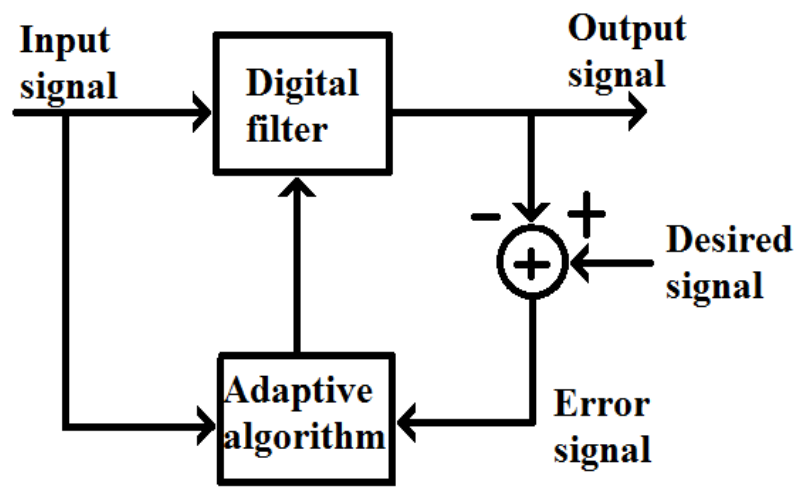

Fig 5: Adaptive filter

Fig 5 shows the basic adaptive filter with input signal and desired signal as inputs and one output signal with adaptive algorithm to adapt changes in the input signal.

\subsubsection{Adaptive filter as noise canceller}

An adaptive filter consists of two distinct parts: a digital filter with adjustable coefficients, and an adaptive algorithm which is used to adjust or modify the coefficients of the filter.

Adaptive noise canceller has two inputs namely primary input $y_{k}$ and reference input $x_{k}$. The primary input receives the signal $s_{k}$ form the signal source that is corrupted by noise $n_{k}$ which is uncorrelated with the signal and the reference input receives noise from the noise source which is uncorrelated with the signal in primary input while correlate with noise in the primary input.

The noise passes through the filter to produce an output $\widehat{n_{k}}$ which is close to the estimate of the primary input noise $n_{k}$. The noise estimate is then subtracted from the corrupted signal to produce an estimate of the signal $\widehat{S_{k}}$ as the output of adaptive noise canceller.

$$
\widehat{s_{k}}=y_{k}-\widehat{n_{k}}=s_{k}+n_{k}-\widehat{n_{k}}
$$

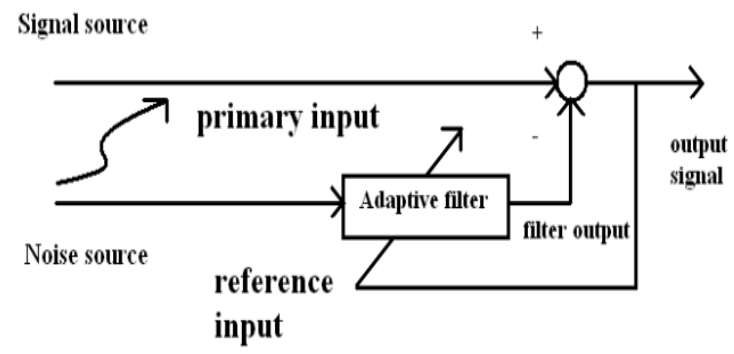

Fig 6: Block diagram of adaptive filter as noise canceller

The main objective in noise cancelling is to produce an optimum estimate of the noise in the contaminated signals and hence an optimum estimates of the desired signal [5].

This is achieved by using error or estimated signal $\widehat{s_{k}}$ in a feedback arrangement to adjust the digital filer coefficients, via a suitable adaptive algorithm, to minimize the noise in $\widehat{s_{k}}$ as shown in fig 6 .

The output signal $\widehat{s_{k}}$ serves two purposes:

1) As an estimate of the desired signal and

2) As an error signal which is used to adjust the filter coefficients.

\section{ADAPTIVE ALGORITHMS}

Adaptive algorithms are used to adjust the coefficients of the digital filter. Such that the error signals $e_{k}$ is minimized according to some criterion.

The most commonly used adaptive algorithms are least mean square (LMS), normalized least mean square (NLMS) because of their robustness and simplicity and also have recursive least square (RLS) algorithm for noise cancellation.

\subsubsection{Least mean square (LMS) algorithm}

One of the most successful adaptive algorithms is the LMS algorithm. Instead of computing $W_{\text {opt }}$ in one go as suggested by wiener-Hopf equation, in the LMS the coefficients are adjusted from sample to sample in such or way as to minimize the MSE [2].

This amounts to descending along the surface of the figure 4 towards its bottom.

An adaptive filter is required to find a solution for its tapweight vector that satisfies the normal equation. An alternative procedure is to use the method of steepest descent, which is one of the oldest methods of optimization.

The standardLMS algorithm performs the following operations to update the coefficients of an adaptive filter:

Calculates the output signal from the adaptive filter.

Calculates the error signalby using the following equation

$$
e_{k}=y_{k}-\widehat{n_{k}}=y_{k}-\sum_{i=0}^{N-1} w(i) \cdot x_{k-i}
$$

Updates the filter coefficients by using the following equation: $W_{k+1}=W_{k}+2 \mu e_{k} x_{k}$

Where $\mu$ is the step size of the adaptive filter, $W_{k}$ is the filter coefficients vector, and $x_{k}$ is the filter input vector.

The LMS algorithm is based on the steepest descent algorithm where the weight vector is updated from sample to sample is shown in figure 7 is as follows

$$
W_{k+1}=W_{k}-\mu \nabla_{k}
$$

Where $W_{k}$ and $\nabla_{k}$ are the weight and the true gradient vectors respectively at the $k^{\text {th }}$ sampling instant. $\mu$ controls the size of incremental correction applied to the adaptive filter from one iteration to the next.

The steepest descent algorithm in the above equation still requires knowledge of $\mathrm{R}$ and $\mathrm{P}$. since $\nabla_{k}$ is obtained by evaluating the equation.

$$
\nabla=\frac{d \xi}{d w}=0-2 P+2 R W
$$

The LMS algorithm is a practical method of obtaining estimates of the filter weights $W_{k}$ in real time without matrix inversion in the equation $W_{o p t}=R^{-1} P$ or the direct computation of the auto correlation and cross correlation.

$$
\nabla=-2 P+2 R W
$$

In the LMS algorithm, instantaneous estimates are used for $\nabla$. Thus

$$
\begin{gathered}
\nabla_{k}=-2 P+2 R W \\
\nabla_{k}=-2 x_{k} y_{k}+2 x_{k} x_{k}^{T} W_{k} \\
\nabla_{k}=-2 x_{k}\left(y_{k}-x_{k}^{T} W_{k}\right) \\
\nabla_{k}=-2 x_{k} e_{k}
\end{gathered}
$$


Where $e_{k}=y_{k}-x_{k}^{T} W_{k}$

Replace the value of $\nabla_{k}$ in steepest descent algorithm then

$$
\begin{gathered}
W_{k+1}=W_{k}-\mu \nabla_{k} \\
W_{k+1}=W_{k}-\mu\left(-2 x_{k} e_{k}\right) \\
W_{k+1}=W_{k}+2 \mu e_{k} x_{k}
\end{gathered}
$$

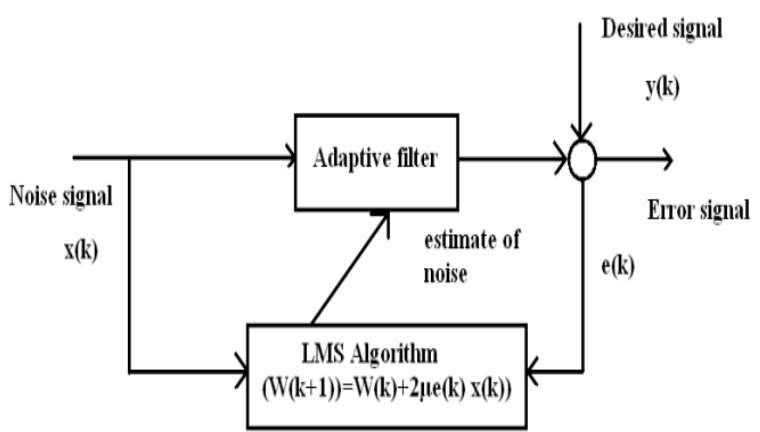

Fig 7: Adaptive filter using LMS algorithm

Clearly, the LMS algorithm above doesn't require prior knowledge of the signal statistics (that is the correlations $\mathrm{R}$ and $\mathrm{P}$ ), but instead uses their instantaneous estimates as shown above.

The weights obtained by the LMS algorithm are only estimates, but these estimates improve gradually with time as weights are adjusted and the filter learns the characteristics of the signals. Eventually, the weights converge [9]

The condition of convergence is

$$
0<\mu>2 / \lambda_{\operatorname{Max}}
$$

Where $\lambda_{\operatorname{Max}}$ is the maximum Eigen value of the input data covariance matrix.

In general $\mathrm{W}_{\mathrm{k}}$ never reaches the theoretical optimum (the wiener solution), but fluctuates about it as shown in figure 8 .

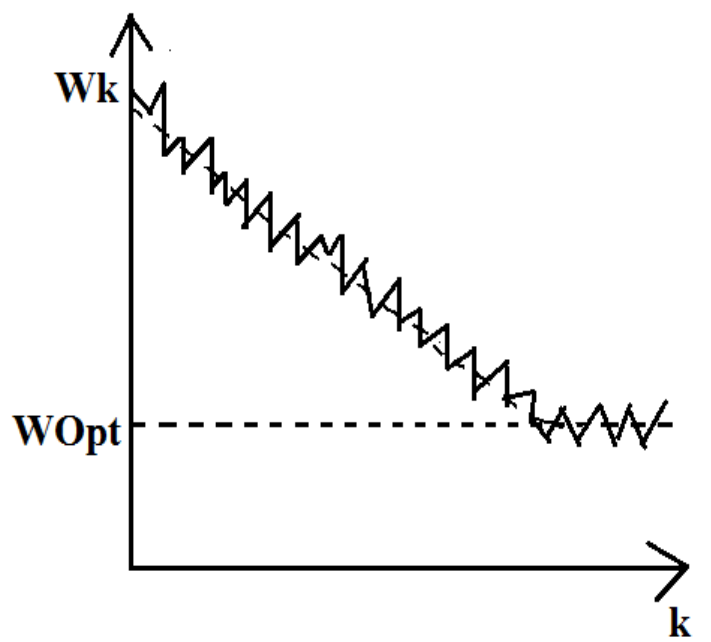

Fig 8: An illustration of the variations in the filter weights

The simplicity of the LMS algorithm and ease of implementation, make it the algorithm of first choice in many real-time systems [10]. The LMS algorithm requires approximately $2 \mathrm{~N}+1$ multiplications and $2 \mathrm{~N}+1$ addition for each new set of input and output samples [7].

In general any signal processing operations involves large no of multiply and accumulate arithmetic operations, because of direct implementation LMS algorithm is more attractive.

\section{REAL TIME ENVIRONMENT}

The proposed algorithm is going to work in real time environment like recorded speech used as input signal and this input signal is going to mixes with the unwanted noise which is also in recorded form and the wiener filter and adaptive algorithms are designed in such a way that these unwanted noise is removed at the end of the final iteration.

Mat lab is the software tool that is going to work on different signal processing tools.

The instruction used in mat lab to record the real time speech signal is given as follows

Wavrecord it Record sound using PC-based audio input device.

Syntax:

Wavrecord (n, fs, 'dtype') uses the data type specified by the string 'dtype' to record the sound.

The string 'dtype' can be one of the following:

'double' (default value), 16 bits/sample

'Single',16 bits/sample

'int16', 16 bits/sample

'uint8', 8 bits/sample

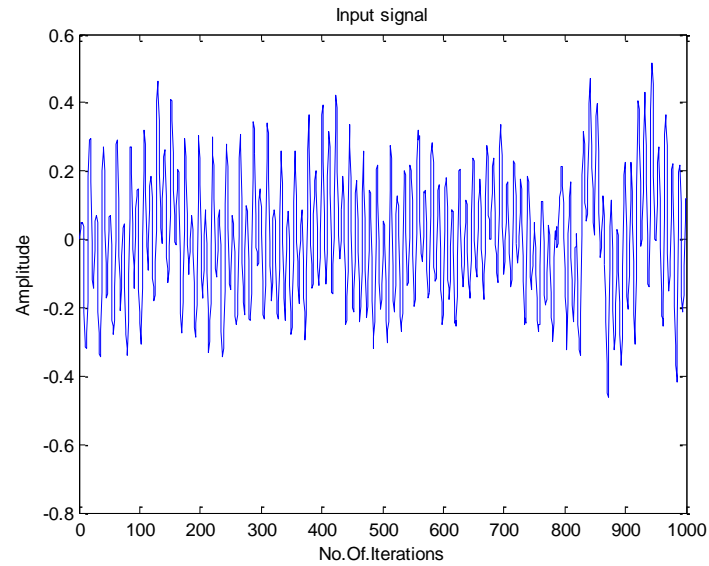

Fig 9: Recorded speech signal

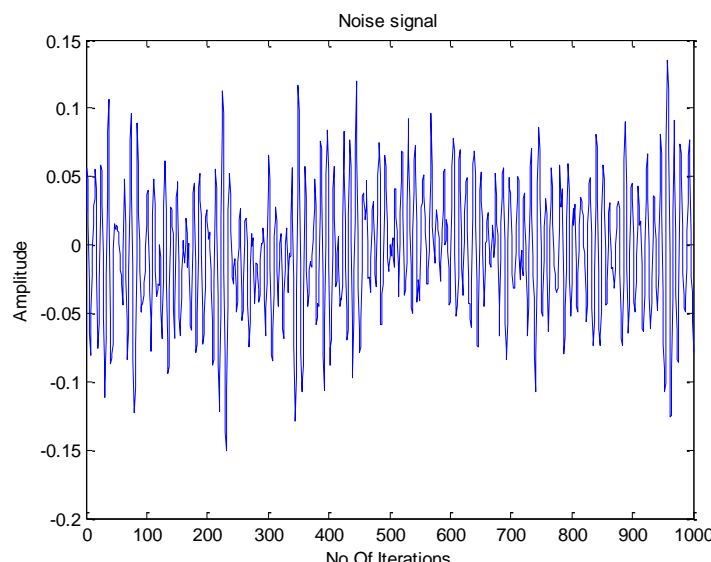

Fig 10: Recorded noise signal

The real time signal that is recorded by using micro phone is shown in figure 9. This signal is going to mix with the recorded unwanted noise signal shown in figure 10 and the 
resultant of the mixing is shown in figure 11. And the mixed noise is eliminated as possible asby using different algorithms at the end of the final iteration.

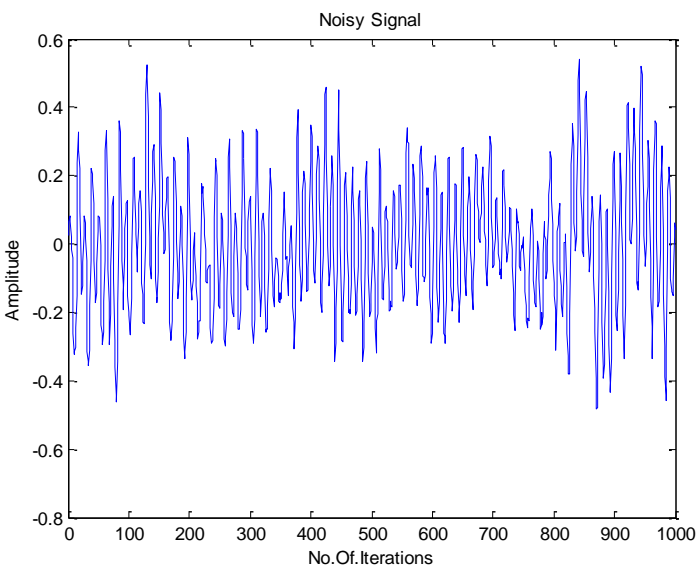

Fig 11: Noisy signal

\section{SIMULATION RESULTS}

The result analysis from the outputs of wiener filter and LMS algorithm shows that Wiener algorithm provides better performance but it has high degree of Complexity.

From the figure 12 it is clear that wiener filter gives good performance but it has high computational complexity. Wiener filter has to compute $\mathrm{R}$ and $\mathrm{P}$ explicitly but LMS algorithm is suited for real time applications where sample by sample bias is done. In wiener filter the computational complexity is also high because of auto correlation and cross correlation so adaptive filters are designed for moderate computations.

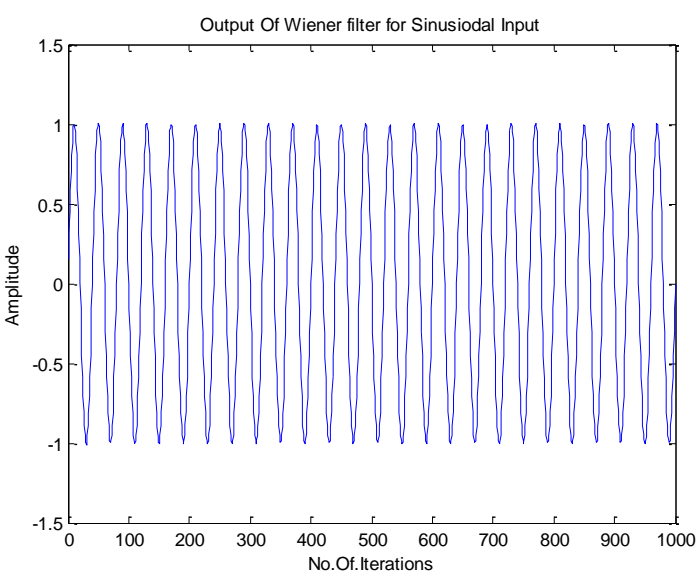

Fig 12: wiener filter output for sinusoidal input

Figure 12 and 13 shows the outputs of wiener filter and LMS algorithm for sinusoidal input. From the figures it is clear that wiener filter will provide better results than LMS algorithm but it requires more complexity.

Whereas LMS algorithm gives better performance for more no of iterations. The output of LMS algorithm is effective when there are large numbers of iterations and also it has low complexity.

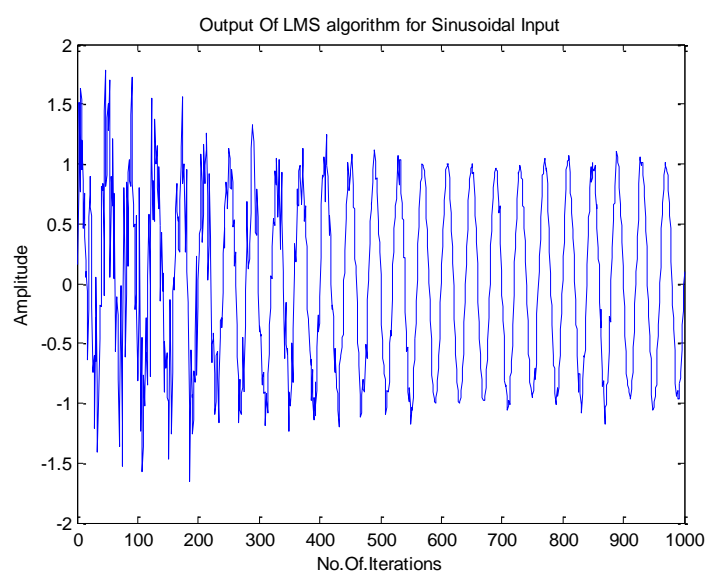

Fig 13: LMS algorithm output for sinusoidal input

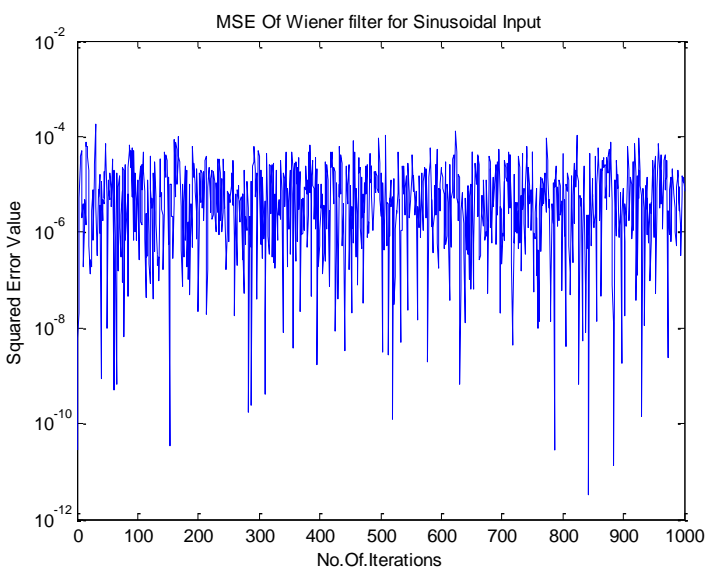

Fig 14: MSE for wiener filter for sinusoidal input

Figure 14 and 15 shows the Mean Square Error for sinusoidal input for wiener filter and LMS algorithm respectively. Figures show that LMS algorithm has Minimum Mean Square Error which yields better results. MSE is decreasing function for LMS algorithm where as in wiener filter MSE is a random function it decreases first and then increases hence LMS algorithm is suitable for better performance and low complexity.

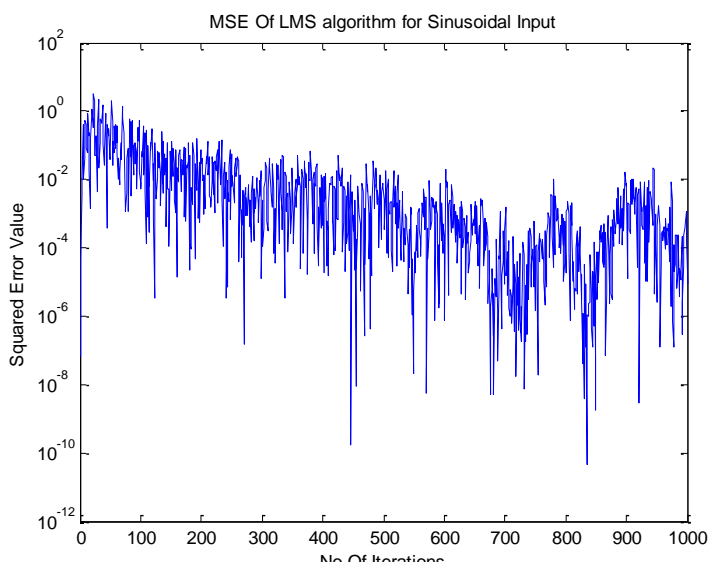

Fig 15: MSE for LMS algorithm for sinusoidal input 


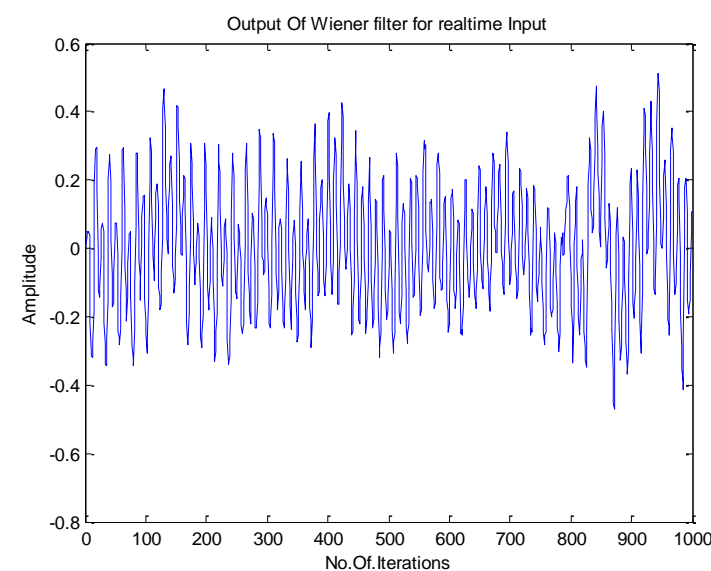

Fig 16: wiener filter output for real time input

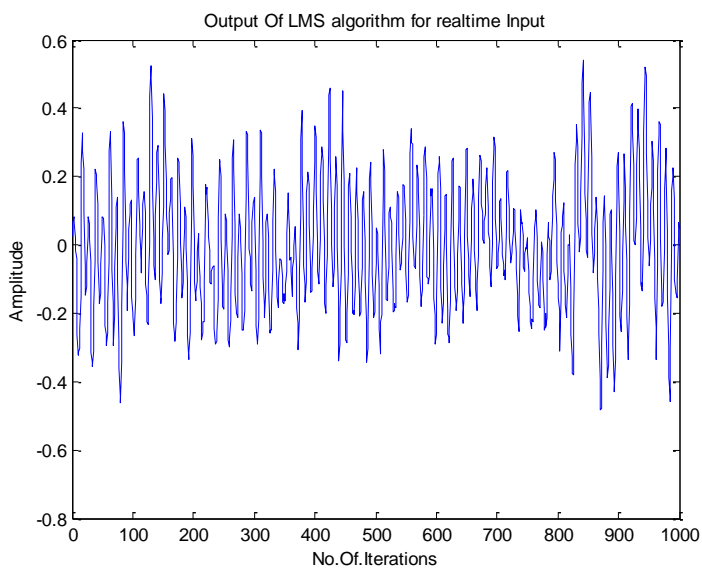

Fig 17: LMS algorithm output for real time input

Figure 16 and 17 shows the outputs of wiener filter and LMS algorithm for real time recorded speech. Wiener filter gives approximately desired signal as output but it has to compute autocorrelation, cross correlation and matrix inversion to yield results whereasLMS algorithm provides alternate solution to optimal filter coefficients problem and removes noise better than wiener filter.

MSE is used to analyze the performance by minimizing it. Mean square error should be as minimum as possible for obtaining good performance of any system.

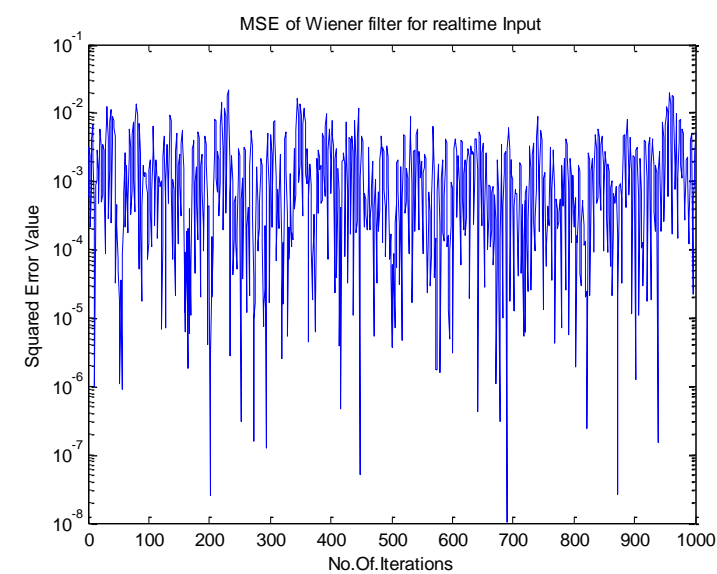

Fig 18: MSE for wiener filter in real time environment

Figure 18 shows the mean square error for wiener filter. From the figure we can analyze that the mean square error increases up to some iteration and after some point MSE is going to decrease. This is the good scenario but it is going to decrease up to some other iteration and will suddenly increase at final iteration.

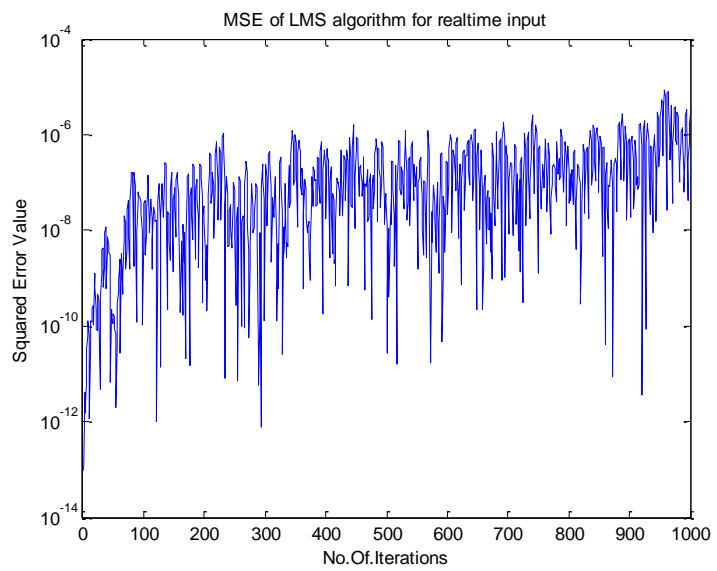

Fig 19: MSE for LMS algorithm in real time environment

Fig 19 shows that the mean square error for LMS algorithm. Figure shows that MSE is an increasing function for this reason the output of LMS algorithm is somewhat distorted. On comparing it with the previous figure both are quite opposite to each other. Though it provides some distorted results than wiener filter it is preferred because of low complexity and convergence rate.

Considering all these parameters wiener filter and LMS algorithms are compared with respect to speed, convergence rate, complexity and mean square error as shown in table 1.

Table 1: Comparison of wiener and LMS algorithm

\begin{tabular}{|c|c|c|c|c|}
\hline Algorithm & Speed & $\begin{array}{c}\text { Converge } \\
\text { nce rate }\end{array}$ & Complexity & MSE \\
\hline $\begin{array}{c}\text { Wiener } \\
\text { filter }\end{array}$ & Low & - & High & Low \\
\hline LMS & High & Low & Low & High \\
\hline
\end{tabular}

\section{CONCLUSION AND FUTURE SCOPE}

Different algorithms have been introduced by several authors to remove noise within the signal and compared their performance with respect to different parameters. Out of which wiener filter is the ancient method for noise cancellation but it requires high complexity. So adaptive filters are introduced to reduce the complexity and computational speed. As the authors tried to reduce the mean square error the complexity and stability of the systems will increases.From the simulation results it is clear that LMS algorithm is best suited because of its low cost and complexity than wiener filter.LMS algorithm is the best algorithm but it has low convergence speed. This project compares only LMS algorithm and wiener filters for real time input. As a future work the same work is going to extend in real time environment with different background noise and compare their performance with other adaptive algorithms i.e., NLMS and RLS to achieve high convergence rate. The performance is analyzed with different parameters and lengths of input signal.

\section{REFERENCES}

[1] Yen-Hsiang chen, Shanq-Jang Ruan, Tom Qi, "An 
Automotive Application of real time adaptive wiener filter for noise cancellation in a car environment," IEEE,2012,4673-2193.

[2] H.Kaur and R.Talwar, "Performance and Convergence Analysis of LMS Algorithm,” IEEE ICCIC, Dec.2012.

[3] Kaur. H and Talwar.R, "Performance comparison of adaptive algorithms for noise cancellation", Engineering trends in communication, C2SPCA 2013.

[4] G.V.P.Chandra Sekhar Yadav and Dr. B. Ananda Krishna, "Study of different adaptive filter algorithms for noise cancellation in real time environment", International journal of computer applications (0975887), vol. 96, no. 10, June. 2014.

[5] B.Widrow and S.D.Stearns, Adaptive Signal Processing, Englewood Cliffs, NJ: Prentice- Hall, 1985.

[6] S.Haykin, Adaptive Filter Theory, Fourth edition, Upper saddle River, NJ: Prentice -Hall, 2002.

[7] J.Gorriz and J.Ramrez, "A Novel LMS Algorithm Applied to Adaptive Noise Cancellation," IEEE Signal Process Letters, vol. 16, no. 1, Jan. 2009.

[8] K. A. Lee, W. S. Gan, and S. M. Kuo, Subband Adaptive Filtering: Theory and Implementation. Hoboken, NJ: Wiley, 2009.

[9] C.Gabriela and M.Sarachin, "Echo Cancellation Using LMS Algorithm," U.P.B Sci Bull., Series C, vol. 71, no. 4, 2009.

[10] B.Widrow， J.R.Glover "Adaptive Noise Cancelling: Principles and Applications," IEEE Proceedings, Vol-63, No.12, Dec. 1975.

[11] Darcy Tsai, Introduction of Wiener Filter, Graduate Institute of Electronics Engineering Nation Taiwan University, Taipei, Taiwan, ROC. 